\title{
ON A CONJECTURE OF H. J. GODWIN ON CUBIC UNITS
}

\author{
VEIKKO ENNOLA
}

\section{Introduction}

Let $K$ be a totally real cubic number field of discriminant $D$. Let $E$ denote the group of norm-positive units of $K$, and take

$$
E_{+}=\{\varepsilon \in E \mid \text { two of the conjugates of } \varepsilon \text { have absolute value }>1\} \text {. }
$$

For any number $\xi$ in $K$ we define

$$
S(\xi)=\frac{1}{2}\left(\left(\xi-\xi^{\prime}\right)^{2}+\left(\xi^{\prime}-\xi^{\prime \prime}\right)^{2}+\left(\xi^{\prime \prime}-\xi\right)^{2}\right),
$$

where $\xi, \xi^{\prime}, \xi^{\prime \prime}$ are the conjugates of $\xi$. We choose a unit $\lambda \in E \backslash\{1\}$ for which $S(\lambda)$ is least, and then another unit $\mu \in E$, not a power of $\lambda$, for which $S(\mu)$ is least. By Lemma 1 below we may assume that $\lambda$ and $\mu \in E_{+}$, and in the sequel we shall always do so. In ([5], p. 321) Godwin made the following conjecture:

(G) If $S(\varepsilon)>9$ for every $\varepsilon \in E \backslash\{1\}$, then $\lambda, \mu$ is a fundamental pair of units.

Brunotte and Halter-Koch [2] showed that $\lambda$ and $\mu$ generate a subgroup of index $\leqq 4$ in the group $E$. Furthermore, they showed that the index is $\leqq 3$ if $S(\mu)>364$. M.-N. Gras [7] proved that (G) is true if $K / Q$ is cyclic. The main result of the present paper is

Theorem 1. If $D$ is sufficiently large, then $(\mathrm{G})$ is true.

From the proof one can compute an explicit upper bound for $D$ in case (G) is violated. It is therefore conceivable that one could check the remaining cases by means of the existing tables of cubic fields, and thus solve the problem completely.

In the classical paper [1] W. E. H. Berwick constructed three units $\theta_{0}, \theta_{1}, \theta_{2}$ in $E$ so that the absolute value of the $i$ th conjugate of $\theta_{i}$ is least among all elements of $E$ such that the absolute values of the other two conjugates are $<1$. Then $\theta_{i}^{-1} \in E_{+}$ $(i=0,1,2), \theta_{0} \theta_{1} \theta_{2}=1$, and any two of the numbers $\theta_{0}, \theta_{1}, \theta_{2}$ form a fundamental pair of units. In Lemma 5 below we shall give a simple alternative characterization of these units. Our proof of Theorem 1 depends upon a connection between the pair $\lambda, \mu$ and the Berwick units. In particular we shall prove 
Theorem 2. If $D$ is sufficiently large and the order of the conjugates of $K$ has been suitably chosen, then either $\lambda=\theta_{0}^{-1}$ or $\lambda=\theta_{0}^{-1} \theta_{1}$.

For any number $\xi$ in $K$, let

$$
S_{*}(\xi)=\xi^{2}+\xi^{\prime 2}+\xi^{\prime \prime 2} .
$$

Define $\lambda_{*}, \mu_{*}$ in the same way as $\lambda, \mu$ but using $S_{*}$ instead of S. Cusick [3] conjectured that $\lambda_{*}, \mu_{*}$ is always a fundamental pair of units, and Godwin [6] proved this conjecture. We shall give yet another proof of Cusick's conjecture by deriving the following close relation between the pair $\lambda_{*}, \mu_{*}$ and the Berwick units:

Theorem 3. If the order of the conjugates of $K$ is suitably chosen, then $\lambda_{*}=\theta_{0}^{-1}$ and $\mu_{*}=\theta_{1}^{-1} \theta_{0}^{k}$ for some non-negative integer $k$.

Additional notation. For any number $\xi$ in $K$ we write $\operatorname{Tr}(\xi)=\xi+\xi^{\prime}+\xi^{\prime \prime}$, $D(\xi)=\left(\xi-\xi^{\prime}\right)^{2}\left(\xi^{\prime}-\xi^{\prime \prime}\right)^{2}\left(\xi^{\prime \prime}-\xi\right)^{2}$, and $M(\xi)=\max \left\{|\xi|,\left|\xi^{\prime}\right|,\left|\xi^{\prime \prime}\right|\right\}$.

If $g \geqq 0$, then $f=O(g)$ and $f \ll g$ both mean that $|f| \leqq C g$ for some absolute positive constant $C$. The symbol $\sim$ means asymptotic equality for $D \rightarrow \infty$.

\section{The auxiliary lemmas}

Lemma 1. Let $\xi \in E_{+}$.

(i) We have $S\left(\xi^{-1}\right) \geqq S(\xi)$ with equality only when $\operatorname{Irr}(\xi, Q)=x^{3}-s x^{2}-(s+3) x-1$ for some integer $s \geqq-1$.

(ii) We have $S_{*}\left(\xi^{-1}\right)>S_{*}(\xi)$.

Proof. Let $f(x)=\operatorname{Irr}(\xi, Q)=x^{3}-s x^{2}+q x-1$. Since

$$
f(1) f(-1)=-\left(1-\xi^{2}\right)\left(1-\xi^{\prime 2}\right)\left(1-\xi^{\prime \prime 2}\right),
$$

the condition $\xi \in E_{+}$is equivalent to $f(1) f(-1)<0$. Clearly, $-f(1) f(-1)=$ $S_{*}\left(\xi^{-1}\right)-S_{*}(\xi)$, whence (ii) follows. Further,

$$
S\left(\xi^{-1}\right)-S(\xi)=(-s+q)(s+q+3)=-f(1) f(-1)-s+q,
$$

so that $S\left(\xi^{-1}\right)>S(\xi)$ if $s \leqq q$. For $s>q$ we must have $-f(-1)=s+q+2<0$, and (i) follows easily.

We find from Lemma 1 that $\lambda, \mu, \lambda_{*}, \mu_{*} \in E_{+}$save that there is a free choice between a unit and its reciprocal in the exceptional case. The choice is made so that $\lambda, \mu \in E_{+}$also in that case.

Lemma 2. For any $\xi \in E \backslash\{1\}$ we have

$$
D \leqq \min \left\{\frac{8}{27} S(\xi)^{3}, 4 M(\xi)^{6}\left(1-M(\xi)^{-6}\right)^{2}\right\} .
$$


Proof. From the inequality of the arithmetic and geometric means we have

$$
D \leqq D(\xi) \leqq(2 S(\xi) / 3)^{3} .
$$

In order to prove the second estimate suppose first that $\xi \in E_{+}$. Let $x$ and $y$ denote those conjugates of $\xi$ which have absolute value $>1$. Then $D(\xi)=f(x, y)$ where

$$
f(x, y)=(x-y)^{2}\left(x-x^{-1} y^{-1}\right)^{2}\left(y-x^{-1} y^{-1}\right)^{2} .
$$

Put $M=M(\xi)$. It is a trivial task to investigate the function $f(x, y)$ in the set $\left\{(x, y) \in \boldsymbol{R}^{2}|1 \leqq| x|\leqq M, 1 \leqq| y \mid \leqq M\right\}$ and to check that the maximum value is $4 M^{6}\left(1-M^{-6}\right)^{2}$.

Suppose next that $\xi \in E \backslash E_{+}$. If, e.g., $|\xi|=M$, then $\left|\xi^{\prime}\right|^{-1}=\left|\xi \xi^{\prime \prime}\right|<M$, $\left|\xi^{\prime \prime}\right|^{-1}=\left|\xi \xi^{\prime}\right|<M$, and it follows from what we have already proved that

$$
D \leqq D\left(\xi^{-1}\right) \leqq 4 M^{6}\left(1-M^{-6}\right)^{2} .
$$

Lemma 3. If $D \geqq 5184$, then $S\left(\xi^{k}\right)>S(\xi)$ for every $\xi \in E \backslash\{1\}$ and every integer $k>1$.

Proof. Lemma 2 implies that $M(\xi)^{3}>36$ and $S(\xi)>25$. From ([5], Lemma 3) we now have

as asserted.

$$
S\left(\xi^{k}\right)>2 \cdot 3^{-k-1} \cdot 25^{k-1} S(\xi)>S(\xi),
$$

Lemma 4. Suppose that $\xi \in E_{+}$and choose the order of the conjugates of $\xi$ so that $\left|\xi^{\prime \prime}\right|<1$. Then

$$
S(\xi)=\left(\xi^{2}-\xi \xi^{\prime}+\xi^{\prime 2}\right)\left(1+O\left(M(\xi)^{-2}\right)\right) .
$$

Proof. For future reference we record here the following obvious inequalities valid for any real $x, y$ :

Since

$$
\max \left\{\frac{3}{4} x^{2}, \frac{3}{4} y^{2}, \frac{1}{2}\left(x^{2}+y^{2}\right)\right\} \leqq x^{2}-x y+y^{2} \leqq \frac{3}{2}\left(x^{2}+y^{2}\right) .
$$

$$
S(\xi)=\xi^{2}+\xi^{2}+\xi^{-2} \xi^{\prime-2}-\xi^{-1}-\xi^{\prime-1}-\xi \xi^{\prime},
$$

the assertion follows immediately from (1).

Lemma 5. Let $\varepsilon_{0}, \varepsilon_{1}, \varepsilon_{2}$ be units in $E_{+}$such that $\varepsilon_{0} \varepsilon_{1} \varepsilon_{2}=1$. If $\varepsilon_{0}, \varepsilon_{1}, \varepsilon_{2}$ generate $E$, then $\varepsilon_{0}^{-1}, \varepsilon_{1}^{-1}, \varepsilon_{2}^{-1}$ are the Berwick units.

Proof. Since $\varepsilon_{0}, \varepsilon_{1}, \varepsilon_{2} \in E_{+}$and $\varepsilon_{0} \varepsilon_{1} \varepsilon_{2}=1$, it is easy to see that for $j \neq k$ the same conjugates of $\varepsilon_{j}$ and $\varepsilon_{k}$ cannot both have absolute value $<1$. Therefore, we may choose the notation so that $\left|\varepsilon_{0}\right|<1,\left|\varepsilon_{1}^{\prime}\right|<1,\left|\varepsilon_{2}^{\prime \prime}\right|<1$. We contend that $\theta_{0}=\varepsilon_{0}^{-1}$. Suppose on the contrary that $\left|\theta_{0}\right|<\left|\varepsilon_{0}\right|^{-1}$. Let $\theta_{0}=\varepsilon_{0}^{a} \varepsilon_{1}^{b}$ for some integers $a, b$. Clearly $a b \neq 0$. We may assume that $b>0$, otherwise we write $\theta_{0}=\varepsilon_{0}^{a-b} \varepsilon_{2}^{-b}$ and 
change the order of the conjugates correspondingly. Now $a>0$ would imply $\left|\theta_{0}^{\prime \prime}\right|>1$, and $a<0$ would imply $\left|\theta_{0}\right|>\left|\varepsilon_{0}\right|^{-1}$, which are both impossible.

Lemma 6. Let $\operatorname{Irr}(\varepsilon, Q)=x^{3}+(l-1) x^{2}-l x-1$ where $l$ is an integer $\geqq 3$, and take $K=\boldsymbol{Q}(\varepsilon)$.

(i) The regulator of the units $\varepsilon, \varepsilon-1$ is $\sim(\ln l)^{2}$.

(ii) We have $S(\varepsilon)=S(\varepsilon-1)=S(\varepsilon+l)=l^{2}+l+1$, and $S(\xi / \eta)>l^{2}+l+1$ for any two distinct $\xi, \eta \in\{\varepsilon, \varepsilon-1, \varepsilon+l\}$.

(iii) If $\varepsilon, \varepsilon-1$ is a fundamental pair of units in $K$, then $\varepsilon^{-1},(\varepsilon-1)^{-1},(\varepsilon+l)^{-1}$ are the Berwick units.

Proof. It is easy to see that the conjugates of $\varepsilon$ lie in the following intervals:

$$
-l^{-1}<\varepsilon<0, \quad 1<\varepsilon^{\prime}<1+l^{-1}, \quad-l<\varepsilon^{\prime \prime}<-l+l^{-2} .
$$

Moreover, one can verify that $-\varepsilon \sim \varepsilon^{\prime}-1 \sim l^{-1}$. Thus

and (i) follows.

$$
\left|\begin{array}{ll}
\ln |\varepsilon| & \ln \left|\varepsilon^{\prime}\right| \\
\ln |\varepsilon-1| & \ln \left|\varepsilon^{\prime}-1\right|
\end{array}\right| \sim(\ln l)^{2},
$$

The assertion (ii) can be proved by a trivial direct computation. To prove (iii) we note that $\varepsilon(\varepsilon-1)(\varepsilon+l)=1$, and that $\varepsilon, \varepsilon-1, \varepsilon+l \in E_{+}$, by (2). The result then follows from Lemma 5 .

Lemma 7. Let $\operatorname{Irr}(\varepsilon, Q)=x^{3}-k x^{2}-(k+3) x-1$, where $k$ is a positive integer, and take $K=Q(\varepsilon)$. Then $K / Q$ is cyclic.

(i) The regulator of the units $\varepsilon, \varepsilon^{\prime}$ is $\sim(\ln k)^{2}$.

(ii) We have $S(\varepsilon)=S\left(\varepsilon^{\prime}\right)=S\left(\varepsilon^{\prime \prime}\right)=k^{2}+3 k+9$, and $S(\xi / \eta)>k^{2}+3 k+9$ for any two distinct $\xi, \eta \in\left\{\varepsilon, \varepsilon^{\prime}, \varepsilon^{\prime \prime}\right\}$.

(iii) If $\varepsilon, \varepsilon^{\prime}$ is a fundamental pair of units in $K$, then $\varepsilon^{-1}, \varepsilon^{\prime-1}, \varepsilon^{\prime \prime-1}$ are the Berwick units.

Proof. Since the discriminant of $\operatorname{Irr}(\varepsilon, Q)$ is $\left(k^{2}+3 k+9\right)^{2}, K / Q$ is cyclic. The conjugates of $\varepsilon$ lie in the intervals

$$
-k^{-1}<\varepsilon<0, \quad-1-k^{-1}<\varepsilon^{\prime}<-1, \quad k+1<\varepsilon^{\prime \prime}<k+1+2 k^{-1} .
$$

Otherwise the argument is similar to that used in the proof of the previous lemma.

Lemma 8. Let $\operatorname{Irr}(\varepsilon, \boldsymbol{Q})$ be one of the polynomials

$$
\begin{aligned}
& f_{1}(x)=x^{3}-k x^{2}+(k-1) x-1, \\
& f_{2}(x)=x^{3}-k x^{2}+(k+1) x-1, \\
& f_{3}(x)=x^{3}-k x^{2}-(k+1) x-1, \\
& f_{4}(x)=x^{3}-k x^{2}-(k+3) x-1,
\end{aligned}
$$


where $k$ is an integer. Suppose that the regulator of $K=\boldsymbol{Q}(\varepsilon)$ is $\sim(\ln |k|)^{2}$. Then, for sufficiently large $|k|$, we have $S(\xi / \eta)>S\left(\theta_{0}^{-1}\right)=S\left(\theta_{1}^{-1}\right)=S\left(\theta_{2}^{-1}\right)$ for any two distinct $\xi, \eta \in\left\{\theta_{0}, \theta_{1}, \theta_{2}\right\}$.

Proof. Put $f(x)=\operatorname{Irr}(\varepsilon, Q)$. We may assume that $|k|$ is large enough.

First, let $f(x)=f_{1}(x)$. If $k<0$, we get $f(x)=x^{3}+(l-1) x^{2}-l x-1$ where $l=-k+1$. It follows from the assumption about the regulator of $K$ and Lemma 6 (i) that $\varepsilon, \varepsilon-1$ is a fundamental pair of units for large $|k|$. The result now follows from Lemma 6 (iii) and (ii). If $k>0$, we have $\operatorname{Irr}(\varepsilon /(\varepsilon-1), Q)=x^{3}+(l-1) x^{2}-l x-1$ where $l=k-4$, and get the same conclusion.

In the next two cases the argument is similar. We have $\operatorname{Irr}(\delta, Q)=x^{3}+(l-1) x^{2}-$ $l x-1$ for a suitable $\delta \in K$ and large positive integer $l$ as follows

$$
\begin{aligned}
& f(x)=f_{2}(x), k<0: \quad \delta=\varepsilon^{-1}, \quad l=-k ; \\
& f(x)=f_{2}(x), k>0: \quad \delta=(1-\varepsilon)^{-1}, \quad l=k-3 \text {; } \\
& f(x)=f_{3}(x), k<0: \quad \delta=\varepsilon+1, \quad l=-k-2 ; \\
& f(x)=f_{3}(x), k>0: \quad \delta=\varepsilon^{-1}+1, \quad l=k-1 .
\end{aligned}
$$

Suppose finally that $f(x)=f_{4}(x)$. Again the argument is the same but relies upon Lemma 7 instead of Lemma 6. If $k<0$ we take $\operatorname{Irr}\left(\varepsilon^{-1}, Q\right)=x^{3}+(k+3) x^{2}+$ $k x-1$ which can be written in the required form on replacing $k+3$ by $-k$.

Lemma 9. Let $\xi \in E_{+},|\xi|<1$, and suppose that $\xi$ is not a power of $\theta_{0}$. Then $\xi=\theta_{0}^{-a} \theta_{j}^{b}$ where $a$ and $b$ are positive integers and $j=1$ or 2 .

Proof. Since $\theta_{0}, \theta_{1}$ is a fundamental pair of units, we can write $\xi=\theta_{0}^{-a} \theta_{1}^{b}$ for some integers $a, b$. By assumption, $b \neq 0$. We may suppose that $b>0$, otherwise we pass to the expression $\xi=\theta_{0}^{-a-b} \theta_{2}^{-b}$. If now $a \leqq 0$, we would have $\left|\xi^{\prime \prime}\right|=$ $\left|\theta_{0}^{\prime \prime}\right|^{-a}\left|\theta_{1}^{\prime \prime}\right|^{b}<1$, which is impossible since $\xi \in E_{+}$.

\section{Proof of Theorem 2}

It follows from Lemma 3 that, for large $D, \lambda$ cannot be a nontrivial power in $E$. Therefore, Theorem 2 is an immediate consequence of the following

Lemma 10. Let $\xi$ be as in Lemma 9 with $j=1$.

(i) If $S(\xi) \leqq S\left(\theta_{0}^{-1}\right)$ and $D$ is sufficiently large, then $a=1$.

(ii) If $S(\xi) \leqq S\left(\theta_{1}^{-1}\right)$ and $D$ is sufficiently large, then $b=1$.

(iii) If the assumptions (i) and (ii) both hold, then we also have $\theta_{0}^{-1} \ll 1$ and $\theta_{1}^{-1} \ll 1$.

Proof. Write $x=\theta_{0}^{\prime-1}, y=\theta_{0}^{\prime \prime-1}, u=\theta_{1}^{-1}, v=\theta_{1}^{\prime \prime-1}$. Then $|x|,|y|,|u|,|v|$ are all $>1$. 
(i) We suppose that $S(\xi) \leqq S\left(\theta_{0}^{-1}\right)$ and that $\xi=\theta_{0}^{-a} \theta_{1}^{b}$ where $a \geqq 2$ and $b>0$. Since $\xi \in E_{+}$we must have $|y|^{a}>|v|^{b}$. Put

$$
\begin{aligned}
M & =\min \left\{M\left(\theta_{0}^{-1}\right), M(\xi)\right\} \\
& =\min \left\{\max \{|x|,|y|\}, \max \left\{|x|^{a}|u v|^{b},|y|^{a}|v|^{-b}\right\}\right\} .
\end{aligned}
$$

From Lemma 4 and the assumption $S(\xi) \leqq S\left(\theta_{0}^{-1}\right)$ we obtain

$$
x^{2 a} u^{2 b} v^{2 b}-x^{a} y^{a} u^{b}+y^{2 a} v^{-2 b} \leqq\left(x^{2}-x y+y^{2}\right)\left(1+O\left(M^{-2}\right)\right) .
$$

From (3) and (1) we further infer

$$
\left(|x|^{a}+|y|^{a}\right)|u|^{b} \ll|x y|^{a}|u|^{b} \leqq \frac{1}{2}\left(x^{2 a} u^{2 b} v^{2 b}+y^{2 a} v^{-2 b}\right) \ll x^{2}+y^{2} .
$$

Therefore, $a=2, u^{b} \ll 1$, and $x^{2} y^{2} \ll x^{2}+y^{2}$. For large $D$ we thus have (cf. Lemma 2)

$$
y^{2}>|v|^{b}=M\left(\theta_{1}^{-1}\right)^{b} .
$$

Hence $|y|$ becomes arbitrarily large so that $x \ll 1$. Since $M \leqq|y|$, the right-hand side of (3) is $y^{2}\left(1+O\left(M^{-1}\right)\right)$. On dividing by $x^{2} y^{2}|u|^{b}$ and denoting $q=x^{2} y^{-2}|u|^{b} v^{2 b}$ we obtain from (3)

$$
q+q^{-1}-\operatorname{sgn}\left(u^{b}\right) \leqq x^{-2}|u|^{-b}\left(1+O\left(M^{-1}\right)\right) .
$$

This is clearly possible only if $u^{b}>0$. Thus

$$
(q-1)^{2} q^{-1}+1-x^{-2} u^{-b}=O\left(M^{-1}\right)
$$

which further implies

$$
q=1+O\left(M^{-1 / 2}\right), \quad x= \pm 1+O\left(M^{-1}\right), \quad u^{b}=1+O\left(M^{-1}\right), \quad v^{b} / y= \pm 1+O\left(M^{-1 / 2}\right) .
$$

In particular, $M \sim|y| \sim|v|^{b}$. These results imply the more accurate estimate

$$
x^{2}-x y+y^{2}=y^{2}\left(1 \pm y^{-1}+O\left(y^{-2}\right)\right),
$$

which in turn allows us to replace (4) by an inequality with right-hand side $|y|^{-1}+O\left(y^{-2}\right)$. It now follows that $x=e+\delta / y$, where $e= \pm 1$ and $|\delta| \leqq 1 / 2+$ $O\left(|y|^{-1}\right)$. Hence

$$
\begin{aligned}
\operatorname{Tr}\left(\theta_{0}\right)-e \operatorname{Tr}\left(\theta_{0}^{-1}\right) & =x y+x^{-1}+y^{-1}-e\left(x^{-1} y^{-1}+x+y\right) \\
& =\delta+e-1+O\left(|y|^{-1}\right)=e-1
\end{aligned}
$$

if $D$ is large enough. Put $k=\operatorname{Tr}\left(\theta_{0}\right)$. Then

$$
\operatorname{Irr}\left(\theta_{0}, Q\right)= \begin{cases}x^{3}-k x^{2}+k x-1 & \text { if } e=1, \\ x^{3}-k x^{2}-(k+2) x-1 & \text { if } e=-1 .\end{cases}
$$

This is impossible because both these polynomials are reducible over $\boldsymbol{Q}$. 
(ii) We suppose that $S(\xi) \leqq S\left(\theta_{1}^{-1}\right)$ and that $\xi=\theta_{0}^{-a} \theta_{1}^{b}$ where $a$ and $b$ are positive. From Lemma 4 and (1) we have

(6)

$$
x^{2 a} u^{2 b} v^{2 b} \ll u^{2}+v^{2}
$$

which clearly implies $b=1$.

(iii) In this case $a=b=1$, i.e. $\xi=\theta_{0}^{-1} \theta_{1}$, and it follows immediately from (6) that $x=\theta_{0}^{\prime-1} \ll 1$. It also follows from (6) that either $|u|$ or $|v|$ is bounded. Suppose that, contrary to the assertion, $v=O(1)$. Denote

$$
M=\min \left\{M\left(\theta_{0}^{-1}\right), M\left(\theta_{1}^{-1}\right), M(\xi)\right\} .
$$

Then, for large $D, M=\min \{|y|,|u|\}$. From Lemma 4 we obtain

$$
\left(x^{2} u^{2} v^{2}-x y u+y^{2} v^{-2}\right)^{2}\left(1+O\left(M^{-2}\right)\right) \leqq S\left(\theta_{0}^{-1}\right) S\left(\theta_{1}^{-1}\right) .
$$

Since $S\left(\theta_{0}^{-1}\right)=y^{2}\left(1+O\left(M^{-1}\right)\right)$ and $S\left(\theta_{1}^{-1}\right)=u^{2}\left(1+O\left(M^{-1}\right)\right)$, we get from this inequality on dividing by $x^{2} y^{2} u^{2}$ that

$$
\left(p+p^{-1}-1\right)^{2} \leqq x^{-2}\left(1+O\left(M^{-1}\right)\right),
$$

where $p=x u v^{2} / y$. Clearly $p>0$. Writing the result in the form

$$
\left(1+p^{-2}\right)(p-1)^{2}+1-x^{-2}=O\left(M^{-1}\right)
$$

we can conclude that

$$
p=1+O\left(M^{-1 / 2}\right), \quad x= \pm 1+O\left(M^{-1}\right), \quad u v^{2} / y= \pm 1+O\left(M^{-1 / 2}\right) .
$$

From $S(\xi) \leqq S\left(\theta_{1}^{-1}\right)$ we infer on dividing by $u^{2}$

$$
x^{2} v^{2}\left(1-p^{-1}+p^{-2}\right) \leqq 1+O\left(M^{-1}\right)
$$

so that $v= \pm 1+O\left(M^{-1 / 2}\right)$ and $u / y= \pm 1+O\left(M^{-1 / 2}\right)$. Hence $M \sim|y| \sim|u|$. We now get for the expression (7) the following more accurate estimate:

$$
\begin{aligned}
x^{-2}((1-x / y)(1-v / u)-1)+O\left(y^{-2}\right) & \leqq|y|^{-1}+|u|^{-1}+O\left(|y|^{-3 / 2}\right) \\
& =2|y|^{-1}+O\left(|y|^{-3 / 2}\right),
\end{aligned}
$$

which leads to $x=e+\delta / y$, where $e= \pm 1$ and $|\delta| \leqq 1+O\left(|y|^{-1 / 2}\right)$. As in (i) above we can conclude that

$$
\operatorname{Tr}\left(\theta_{0}\right)-e \operatorname{Tr}\left(\theta_{0}^{-1}\right) \in\{e-2, e-1, e\} .
$$

Therefore, $\operatorname{Irr}\left(\theta_{0}, \boldsymbol{Q}\right)$ must be one of the four polynomials $f_{i}(x)$ in Lemma 8 because the two polynomials in (5) cannot occur. Since $\theta_{0}, \theta_{1}$ is a fundamental pair of units, the regulator of $K$ is

$$
\left|\begin{array}{cc}
\ln |x y|-\ln |x| \\
-\ln |u| & \ln |u v|
\end{array}\right| \sim(\ln |y|)^{2} \sim\left(\ln \left|\operatorname{Tr}\left(\theta_{0}\right)\right|\right)^{2},
$$

and Lemma 8 leads to a contradiction for large $D$. 


\section{Proof of Theorem 1}

Assuming that $D$ is sufficiently large we know that $\lambda=\theta_{0}^{-1}$ or $\theta_{0}^{-1} \theta_{1}$ if the notation is suitably chosen. We also know from Lemma 3 that $\mu$ cannot be a nontrivial power in $E$.

Consider first the possibility $\lambda=\theta_{0}^{-1}$. We may assume that $\mu^{-1}$ is not one of the Berwick units because otherwise $(\mathrm{G})$ is true. By Lemma $9, \mu=\theta_{i}^{-a} \theta_{j}^{b}$ where $i, j \in\{0,1,2\}, i \neq j$, and $a$ and $b$ are positive integers. If $i=0$ then $b=1$ for large enough $D$, by Lemma 10 . Hence (G) is true. The same conclusion follows if $j=0$. In the remaining case we may assume, e.g., that $i=1, j=2$. From Lemma 10, $a=b=1$, i.e. $\mu=\theta_{1}^{-1} \theta_{2}$. In this case (G) would be violated, but we shall deduce a contradiction.

Write $u=\theta_{1}^{-1}, v=\theta_{1}^{\prime \prime-1}, s=\theta_{2}^{-1}, t=\theta_{2}^{\prime-1}$. Then $|u|,|v|,|s|,|t|$ are all $>1$. By Lemma 10 (iii), we have $v \ll 1, t \ll 1$. Take

$$
M=\min \left\{M\left(\theta_{0}^{-1}\right), M\left(\theta_{1}^{-1}\right), M\left(\theta_{2}^{-1}\right), M(\mu)\right\},
$$

so that $M=\min \{|u|,|s|\}$ if $D$ is sufficiently large. Since $S\left(\theta_{0}^{-1}\right) \leqq \min \left\{S\left(\theta_{1}^{-1}\right), S\left(\theta_{2}^{-1}\right)\right\}$, we obtain from Lemma 4

$$
\left(u^{2} v^{2} t^{-2}-u s+s^{2} t^{2} v^{-2}\right)^{2}\left(1+O\left(M^{-2}\right)\right) \equiv S\left(\theta_{1}^{-1}\right) S\left(\theta_{2}^{-1}\right) .
$$

On substituting $S\left(\theta_{1}^{-1}\right)=u^{2}\left(1+O\left(M^{-1}\right)\right)$ and $S\left(\theta_{2}^{-1}\right)=s^{2}\left(1+O\left(M^{-1}\right)\right)$ in this inequality and dividing by $u^{2} s^{2}$ we get

$$
\left(q+q^{-1}-1\right)^{2} \leqq 1+O\left(M^{-1}\right),
$$

where $q=u v^{2} s^{-1} t^{-2}$. As before, it follows that $q=1+O\left(M^{-1 / 2}\right)$, and therefore

$$
u / s=t^{2} / v^{2}+O\left(M^{-1 / 2}\right) \ll 1 .
$$

From the condition $S(\mu) \leqq S\left(\theta_{2}^{-1}\right)$ we now find that

$$
v^{2} t^{2}-s^{-1} v t(u / s)+s^{-2}\left(u^{2} / s^{2}\right) \leqq 1+O\left(M^{-1}\right),
$$

i.e. $v^{2} t^{2} \leqq 1+O\left(M^{-1}\right)$. Hence

$$
v= \pm 1+O\left(M^{-1}\right), \quad t= \pm 1+O\left(M^{-1}\right), \quad u / s=1+O\left(M^{-1 / 2}\right),
$$

so that $M \sim|u| \sim|s|$. We can now rewrite (8) in the more accurate form

$$
v^{2} t^{2}-|s|^{-1}+O\left(|s|^{-3 / 2}\right) \leqq 1+|s|^{-1}+O\left(s^{-2}\right) .
$$

It follows that $t=e+\delta / s$, where $e= \pm 1$ and $|\delta| \leqq 1+O\left(|s|^{-1 / 2}\right)$, which leads to the same contradiction as at the end of the proof of Lemma 10.

Suppose next that $\lambda=\theta_{0}^{-1} \theta_{1}$. Write $x=\theta_{0}^{\prime-1}, y=\theta_{0}^{\prime \prime-1}, u=\theta_{1}^{-1}, v=\theta_{1}^{\prime \prime-1}$. From Lemma 10 (iii) we have $x \ll 1, u \ll 1$. Put

$$
M=\min \left\{M\left(\theta_{0}^{-1}\right), M\left(\theta_{1}^{-1}\right), M\left(\theta_{2}^{-1}\right), M(\lambda)\right\} .
$$


Obviously, for large $D, M=\min \{|y|,|v|\}$. Let $\mu=\theta_{0}^{a} \theta_{1}^{b}$ for some integers $a, b$. Since $\mu$ is not a power of $\lambda$, we must have $a+b \neq 0$. We can also assume that $a+b \neq \pm 1$, because otherwise (G) is true. It then follows that $a b \neq 0$, by Lemma 3 . We cannot have $a b<0$, because in that case Lemma 10 would imply that $\mu=\lambda$ or $\lambda^{-1}$ if $D$ is large enough.

Suppose first that $a>0, b>0$. The condition $S(\mu) \leqq S\left(\theta_{1}^{-1}\right)$ gives, by (1),

$$
x^{2 a} y^{2 a} u^{-2 b} \ll v^{2}, \quad x^{-2 a} u^{2 b} v^{2 b} \ll v^{2} .
$$

On multiplying these inequalities and taking into account that $|y|>|v|$ because $\lambda \in E_{+}$, we obtain $v^{2(a+b)} \ll v^{4}$. Thus $a=b=1$, i.e. $\mu=\theta_{2}^{-1}$. From $S(\mu)=S\left(\theta_{2}^{-1}\right) \leqq$ $S\left(\theta_{1}^{-1}\right)$ we then find

$$
x^{2} y^{2} u^{-2}-y v+x^{-2} u^{2} v^{2} \leqq v^{2}\left(1+O\left(M^{-1}\right)\right) .
$$

It is easy to see that $y v$ must be positive. On dividing by $y v$ we can write the above inequality in the form

$$
p+p^{-1}-1-v / y \ll M^{-1}
$$

where $p=x^{2} y u^{-2} v^{-1}$. Since $0<v / y<1$, we can apply the same argument as before and deduce that

$$
y / v= \pm 1+O\left(M^{-1}\right), \quad p=1+O\left(M^{-1 / 2}\right), \quad x / u= \pm 1+O\left(M^{-1 / 2}\right) .
$$

Now $S(\lambda) \leqq S\left(\theta_{1}^{-1}\right)$ implies

$$
x^{2} u^{2} v^{2} \leqq v^{2}\left(1+O\left(M^{-1}\right)\right)
$$

whence $x= \pm 1+O\left(M^{-1}\right)$ and $u= \pm 1+O\left(M^{-1}\right)$. Applying (9) in a more accurate form and arguing as before we again conclude that $\operatorname{Irr}\left(\theta_{0}, \boldsymbol{Q}\right)$ is one of the four polynomials in Lemma 8 which is impossible.

Suppose finally that $a<0, b<0$. In this case

$$
v^{4} \leqq y^{-2 a} v^{-2 b} \ll S(\mu) \leqq S\left(\theta_{1}^{-1}\right) \ll v^{2},
$$

which is absurd. This completes the proof of Theorem 1.

\section{Proof of Theorem 3}

We may assume the order of the conjugates of $K$ to be chosen so that $\left|\lambda_{*}\right|<1$. Put $p=\lambda_{*}^{\prime 2}, q=\lambda_{*}^{\prime \prime 2}, x=\theta_{0}^{\prime-2}, y=\theta_{0}^{\prime \prime 2}$. Then $p, q, x, y$ are $>1$, and we have

$$
S_{*}\left(\theta_{0} \lambda_{*}\right)=p^{-1} q^{-1} x y+p x^{-1}+q y^{-1} \text {. }
$$

Suppose that, contrary to the assertion, $\theta_{0} \lambda_{*} \neq 1$, and let $f(x, y)$ denote the righthand side of (10). It follows from the definition of $\theta_{0}$ that necessarily $x y \leqq p q$. Put

$$
A=\left\{(x, y) \in \boldsymbol{R}^{2} \mid x \geqq 1, y \geqq 1, x y \equiv p q\right\},
$$


and consider the function $f(x, y)$ for fixed $p, q$ in the set $A$. It is an elementary task to verify that $f(x, y)$ attains its maximum in $A$ only at the point $(x, y)=(1,1)$. Therefore,

$$
S_{*}\left(\theta_{0} \lambda_{*}\right)<f(1,1)=S_{*}\left(\lambda_{*}\right),
$$

which contradicts the definition of $\lambda_{*}$.

We have thus proved that $\lambda_{*}=\theta_{0}^{-1}$. Consider now $\mu_{*}$. We cannot have $\left|\mu_{*}\right|<1$, because otherwise the above argument would lead to the contradiction $S_{*}\left(\theta_{0} \mu_{*}\right)<$ $S_{*}\left(\mu_{*}\right)$. Interchanging the second and third conjugate if need be we assume that $\left|\mu_{*}^{\prime}\right|<1$. If $\theta_{1} \mu_{*}$ is not a power of $\theta_{0}$, the same argument would give $S_{*}\left(\theta_{1} \mu_{*}\right)<$ $S_{*}\left(\mu_{*}\right)$, contrary to the definition of $\mu_{*}$. Hence $\mu_{*}=\theta_{1}^{-1} \theta_{0}^{k}$. By the definition of $\theta_{1},\left|\mu_{*}^{\prime}\right| \leqq\left|\theta_{1}^{\prime}\right|^{-1}$, i.e. $\left|\theta_{0}^{\prime}\right|^{k} \leqq 1$. Thus $k \geqq 0$, and the proof is complete.

\section{References}

[1] BeRwICK, W. E. H.: Algebraic number-fields with two independent units. - Proc. London Math. Soc. $34,1932,360-378$.

[2] BRUnotte, H., und F. HAlter-Koch: Zur Einheitenberechnung in totalreellen kubischen Zahlkörpern nach Godwin. - J. Number Theory 11, 1979, 552-559.

[3] Cusick, T. W.: Finding fundamental units in cubic fields. - Math. Proc. Cambridge Philos. Soc. 92, 1982, 385-389.

[4] Cusick, T. W.: Finding fundamental units in totally real fields. - Math. Proc. Cambridge Philos. Soc. 96, 1984, 191-194.

[5] Godwin, H. J.: The determination of units in totally real cubic fields. - Proc. Cambridge Philos. Soc. $56,1960,318-321$.

[6] Godwin, H. J.: A note on Cusick's theorem on units in totally real cubic fields. - Math. Proc. Cambridge Philos. Soc. 95, 1984, 1-2.

[7] Gras, M.-N.: Note a propos d'une conjecture de H. J. Godwin sur les unités des corps cubiques. - Ann. Inst. Fourier (Grenoble) 30:4, 1980, 1-6.

[8] HaLter-Koch, F.: Metrische Theorie der Einheiten algebraischer Zahlkörper. - Mitt. Math. Ges. Hamburg 11, 1982, 131-141.

[9] Watabe, M.: On certain cubic fields I, III. - Proc. Japan Acad. Ser. A Math. Sci. 59, 1983, $66-69$ and $260-262$.

University of Turku

Department of Mathematics

SF-20500 Turku

Finland

Received 27 April 1987 